\title{
Linx
}

Revue des linguistes de l'université Paris X Nanterre

$77 \mid 2018$

Regards croisés sur le futur en français et dans différentes langues romanes

\section{Divergence et convergence d'emploi du futur simple et du futur périphrastique}

\section{Marie-Armelle Camussi-Ni}

\section{(2) OpenEdition}

\section{Journals}

Édition électronique

URL : http://journals.openedition.org/linx/2702

DOI : $10.4000 /$ linx.2702

ISSN : 2118-9692

Éditeur

Presses universitaires de Paris Nanterre

\section{Édition imprimée}

Date de publication : 30 octobre 2018

Pagination : 41-64

ISBN : 978-2-84016-329-9

ISSN : 0246-8743

Référence électronique

Marie-Armelle Camussi-Ni, « Divergence et convergence d'emploi du futur simple et du futur périphrastique », Linx [En ligne], 77 | 2018, mis en ligne le 30 avril 2019, consulté le 03 mai 2019. URL http://journals.openedition.org/linx/2702 ; DOI : 10.4000/linx.2702

Ce document a été généré automatiquement le 3 mai 2019.

Département de Sciences du langage, Université Paris Ouest 


\title{
Divergence et convergence d'emploi du futur simple et du futur périphrastique
}

\author{
Marie-Armelle Camussi-Ni
}

\section{Introduction}

1 La question de la concurrence entre futur simple (FS) et futur périphrastique (FP) a été souvent posée, en particulier sous les termes d'une grammaticalisation aboutissant au remplacement du FS par le FP (Fleishman, 1983; Barcelo, 2007). Qu'elle ait été abordée sous l'angle sociolinguistique par l'étude de données de corpus oraux comme par Emirkanian et Sankoff (1986) et par Jeanjean (1988) ou encore sous l'angle de l'analyse sémantique (Franckel, 1984 ; Vet, 1993 ; Laurendeau, 2000 ; Barbazan, 2010), elle a reçu des réponses très différentes.

2 Cette question reste ouverte comme en témoignent les conclusions apparemment opposées de deux études sur corpus de l'oral parues récemment :

3 - S. Fleury et S. Branca-Rosoff (2010), analysant un corpus oral de parisiens natifs, s'appuient sur la comparaison entre les générations pour souligner la persistance du FS, suivant en cela l'hypothèse de Labov.

4 - L. Abouda et M. Skrovec (2015), comparant le corpus d'Orléans original datant des années 1968-1971 (ESLO 1) et le corpus ESLO 2 en cours de réalisation notent un effritement du FS au profit du FP.

5 Il s'agira ici d'explorer la question de l'interchangeabilité du FS et du FP à partir d'analyses morphologique et sémantique du FS de façon à non seulement mettre ces dernières à l'épreuve du contraste avec les emplois du FP mais aussi envisager sous un autre angle la question de l'éventualité du remplacement du FS par le FP.

6 Dans une première partie, nous mettrons notre analyse du FS en perspective avec une analyse du FP. Dans un deuxième temps, sera opéré un classement analytique des 
différents cas de concurrence entre FS et FP selon que leur interchangeabilité semble impossible, peu fréquente ou possible. Enfin, les résultats de cette analyse seront confrontés à celle du FS dans son opposition avec le conditionnel pour examiner si cette dernière s'en trouve confortée.

\section{Mise en perspective d'une analyse originale du FS avec celle du FP}

\subsection{Présence d'un morphème $R$ commun au $F S$, au conditionnel et à l'infinitif}

Il a été montré (Camussi-Ni, 2010) que l'analyse morphologique couramment admise d'un conditionnel constitué d'un suffixe $\mathbf{R}$ du FS et d'un suffixe ai de l'imparfait impliquait nécessairement la mise en œuvre d'une analyse symétrique du FS en deux morphèmes : le $\mathbf{R}$, commun au FS et au conditionnel, et le a qui s'oppose au ai ${ }^{1}$ du conditionnel.

Tu conclu-r-ai-s

Tu conclu-r-a-s

8 Le repérage du premier morphème, $\mathbf{R}$, commun au FS et au conditionnel, conduit à examiner l'hypothèse qu'il serait également commun à l'infinitif. FS et infinitif partageraient alors un morphème $\mathbf{R}$, qu'on pourrait dégager par opposition morphologique, en admettant qu'il présente différents allomorphes² :

prend-re /Øprãd-r-Ø-Ø/

Tu prend-r-a-s /typrãd-r-a- $\varnothing /$

Dans cette analyse, l'infinitif combine à une base lexicale et à un suffixe $\mathbf{R}$ deux zéros ${ }^{3}$ indiquant une absence de marque de personne et de détermination du procès. L'infinitif serait ainsi constitué de vides formels qui autoriseraient tous les réinvestissements sémantiques possibles intervenant de façon contextuelle ou co-textuelle.

Cette analyse s'appuie sur celle, syntaxique, d'Allaire: «Le verbe [infinitif] - avec les silences qu'il convoie - n'a d'existence que par le rôle qui lui est conféré et conféré corrélativement à d'autres termes dans la phrase. Il suffit d'ailleurs que les silences de l'infinitif ne soient pas syntaxiquement exploités par les formes pour que l'incomplétude verbale appelle en performance le complément de la situation. Ainsi s'explique l'énoncé exclamatif 'danser!' (cf. 'Il est si grand!', 'Cette fleur!') ou jussif 'danser en ligne' ${ }^{4}$ (Allaire, 1984: 73). Ainsi, le verbe à l'infinitif ne pourrait-il s'actualiser que dans les relations syntaxiques ou contextuelles qui permettent de remplir les zéros qu'il comporte. En effet, l'absence de personne du verbe à l'infinitif va de pair avec une absence de création de situation d'énonciation de référence et donc une absence de détermination du procès dans une situation d'énonciation.

11 Soulignons d'emblée que le terme "situation d'énonciation » est à comprendre comme « interne à l'énoncé ». Comme le souligne de Vogüé, il y peut y avoir «maldonne » sur ce concept. Ce qu'elle précise à propos du concept d'énonciation dans la théorie culiolienne peut être ici repris: "Il ne désigne pas l'événement singulier réel où un énoncé est proféré, à savoir une situation d'interlocution [...] On distingue entre énonciation et locution, précisément parce que l'on considère que l'énonciatif est interne à la langue ; c'est une configuration complexe de paramètres abstraits (qui, éventuellement, vont 
pouvoir se rapporter aux différentes composantes d'une situation réelle) à partir desquels se construit le calcul référentiel interne à l'énoncé » (de Vogüé, 1991 : 38).

Or, sélectionner un verbe à l'infinitif équivaut à ne pas désigner, du moins par ce verbe, de situation d'énonciation pour l'opération de référence, puisque le repérage d'un sujet par rapport au locuteur est impossible, et de ce fait, celui du procès par rapport à la situation d'énonciation.

C'est ce que souligne aussi ce propos de Rémy-Giraud (1988:14) : «Les formes verbales personnelles sont des formes qui, à la différence des formes verbales non personnelles, contiennent une référence à la situation d'énonciation ». Ce qui signifie qu'inversement, les formes non-personnelles comme l'infinitif ne contiennent pas de référence à la situation d'énonciation.

C'est la liberté de réinvestissement dans une relation à la situation d'énonciation qui peut conduire à attribuer à l'infinitif le sens de "virtualité " ou de "potentialité » de l'accomplissement que l'on peut trouver chez de nombreux auteurs (ainsi, "virtualité » chez Rémy-Giraud (1988), Allaire (1984), «mode des possibles» pour Bres (2009) " potentialité » chez Guillaume (1929), ou encore d'« éventualité » telle que de Vogüé (2006) la définit ${ }^{5}$. Or, selon notre analyse, le morphème $\mathbf{R}$ participe à la désignation de cette valeur que nous nommerons "potentielle " dans la mesure où c'est lui qui donne leur valeur aux zéros avec lesquels il se combine ( $c f$. Camussi-Ni, 2010), c'est lui qui indique qu'il s'agit d'un verbe et que les zéros sont opposables à des marques de personne ou de « temps».

De leur côté, FS et conditionnel qui partagent un suffixe $\mathbf{R}$ désignent tous deux un procès qui n'est pas avéré et qui peut être qualifié de « procès potentiel ».

Aussi est-il possible de postuler qu'infinitif, conditionnel et FS partagent la même valeur sémantique «potentiel » qui s'oppose à "avéré ». Alors que la valeur "potentiel » de l'infinitif prend son sens dans l'absence de référence à une situation d'énonciation, la valeur « potentiel » du FS et du conditionnel s'applique à un procès défini en référence à une situation d'énonciation.

Dans la mesure où le FP se construit avec un verbe à l'infinitif, reconnaitre un morphème commun au FS et à l'infinitif, le $\mathbf{R}$, permet de postuler une valeur commune entre FS et FP, qui justifie de leur proximité, celle que nous avons choisi de nommer " potentiel». Il reste alors à analyser les autres éléments des deux formes : la composition du FP et le morphème $\mathbf{a} \mathrm{du} \mathrm{FS}$.

\subsection{La composition du FP}

Le FP est une construction figée composée du verbe aller et d'un verbe à l'infinitif, porteur du morphème $\mathbf{R}$ et de zéros susceptibles de se réinvestir dans leur relation syntaxique avec le verbe aller. Le figement de la construction s'observe dans :

- la restriction en temps du verbe aller aux seuls présent et imparfait (elle va venir, elle allait venir $/{ }^{*}$ elle alla venir, ${ }^{*}$ elle ira venir, ${ }^{*}$ elle irait venir) qui atteste de la solidarité des deux verbes ;

- sa désémantisation

Selon l'analyse de Vetters et Lière (2009), le verbe aller se serait désémantisé au cours de sa grammaticalisation, passant du sens de déplacement à celui d'intention pour finalement perdre tout sens dans le cadre de la périphrase à l'instar de l'auxiliaire avoir 
dans les formes composées. Les auteurs confortent ainsi le choix de Vet (2007) de placer le FP dans le système des temps verbaux. situation potentielle (partir) est mise en relation avec une situation coïncidente avec la situation d'énonciation (ils vont), elle-même construite par le locuteur. Un verbe à l'infinitif, désignant une situation potentielle, est construit avec un verbe désémantisé désignant un procès coïncident avec la situation d'énonciation dans son ensemble. Le FP mettrait donc en lien le procès potentiel avec les paramètres de la situation d'énonciation.

\subsection{Le FS : combinaison du suffixe $R$ et du suffixe a}

Alors que le FP résulte de la mise en relation grammaticalisée de deux unités verbales, combinant de façon fragmentée la personne, le présent et la valeur «potentiel », le FS se présente comme une seule unité qui combine à la fois la personne, la valeur " potentiel » réinvestissant le $\mathbf{R}$ et une autre valeur sémantique réinvestissant le $\mathbf{a}$.

Ce second morphème du FS mis en évidence par opposition avec le morphème de l'imparfait inclus dans le conditionnel, conduit à deux analyses possibles, si on examine les oppositions dans lesquelles entre le morphème de l'imparfait :

- soit il correspond au morphème du présent, auquel cas, les deux futurs seraient équivalents et la distribution libre quels que soient les contextes, ou tout au moins pas explicable par les oppositions de valeur ;

- soit il correspond à un morphème du passé simple et FP et FS ne se différencieraient pas seulement par leur construction mais surtout par la présence du morphème du présent et celle du morphème du passé simple.

Si la première hypothèse présente l'avantage de correspondre à l'analyse étymologique reconnue du FS (il se serait construit à partir de la périphrase verbale habeo facere incluant un infinitif et le verbe avoir au présent), rien n'empêche de penser qu'il y a eu redistribution des valeurs de temps à l'intérieur du système verbal depuis l'apparition du FS, d'autant que l'absence de FP en ancien français et la réorganisation des emplois entre 
subjonctif et conditionnel entre le $16^{\text {ème }}$ et le $18^{\text {ème }}$ siècle incitent à postuler une telle redistribution.

L'hypothèse d'un morphème a correspondant au passé simple - hypothèse déjà proposée par Guillaume (1929) - peut paraître plus satisfaisante, sur le plan morphologique comme sur le plan sémantique, quand on oppose FS et conditionnel.

Nous cherchons ici à mettre à l'épreuve cette hypothèse à travers la question de la concurrence FP/FS.

Si le FS comportait un morphème du présent, alors FS et FP seraient très proches sémantiquement. Si le FS comportait un morphème du passé simple, alors cela pourrait justifier d'une divergence d'emploi. Aussi est-il nécessaire de revenir sur les cas de divergence, de préférence d'emploi ou encore d'équivalence glosés par les descripteurs des deux futurs.

\section{Examen des différents cas de figure de concurrence FS/FP à travers des exemples}

\subsection{Distribution restreinte des deux futurs en contexte}

\section{A. Restriction d'emploi du FS}

31 Un premier type d'énoncés se prête particulièrement mal à l'interchangeabilité du FP par le FS. Il s'agit d'énoncés où une apostrophe comme «Tiens!» ou «Attention! indique la focalisation du locuteur sur un élément de la situation d'énonciation qui induit un procès potentiel ${ }^{7}$. Cette situation d'énonciation peut être mise en scène dans le co-texte, rendant alors ce sens évident et l'interchangeabilité avec le FS très peu probable :

(1) Ça coule toujours à côté : «Attention, tu vas salir la nappe »

? Ça coule toujours à côté : «Attention, tu saliras la nappe »

(2) Tiens, il va pleuvoir

? Tiens, il pleuvra

(3) Pierre, fais donc attention, tu vas te faire écraser par la voiture qui arrive

? Pierre, fais donc attention tu te feras écraser par la voiture qui arrive

Ces emplois du futur périphrastique sont souvent qualifiés d'«imminents», ce qui signifie qu'on se représente une continuité temporelle entre la situation d'énonciation et la situation du procès potentiel. Pourtant, si le paramètre temporel est indissociable de ces énoncés, ce n'est pas proprement celui qui introduit une continuité entre les deux situations mais bien plutôt l'existence de circonstances qui leur sont communes.

En effet, ces énoncés ont en commun de désigner des circonstances de la situation d'énonciation - sur lesquelles le locuteur attire l'attention - qui sont autant de conditions de réalisation du procès potentiel.

Pour l'énoncé (1), il peut s'agir de la maladresse de l'interlocuteur ou de son inattention, de l'aspect liquide du miel, ou encore de la minceur de la tartine. Pour l'énoncé (2), ce peut être l'assombrissement du ciel, l'intensification de la douleur rhumatismale du locuteur, la malchance habituelle de l'interlocuteur qui vient de déclarer vouloir se promener qui sont implicitement désignés dans Tiens. L'une ou l'autre de ces circonstances de la situation d'énonciation constitue alors une condition du procès potentiel exprimé par le verbe au FP. 
35 Si les paramètres ne sont pas forcément explicitement exposés, ils le sont dans l'énoncé (3) où le contexte donne des indications sur l'élément de la situation d'énonciation en relation avec la situation du procès au FP: la voiture qui arrive dans la situation d'énonciation est celle qui est agent du procès potentiel tu vas te faire écraser. Ainsi, dans ces énoncés où il est indiqué que les paramètres de la situation d'énonciation sont ceux qui conditionnent la réalisation du procès potentiel, l'emploi d'un FP qui associe une référence à la situation d'énonciation à un procès potentiel apparaît contraint. À l'inverse, le FS serait incohérent dans ces énoncés qui exhibent la communauté de paramètres de la situation d'énonciation et de la situation du procès potentiel. C'est donc qu'il introduit une valeur discordante entre les deux situations.

\section{B. Restriction d'emploi du FP}

37 Symétriquement, la sélection du FS peut être la seule envisageable quand le contexte implique une absence de paramètres communs entre la situation d'énonciation et la situation du procès potentiel. C'est le cas de certains énoncés au FS mis en relation avec une hypothèse :

(4) Si je réussis le concours, je prendrai des vacances.

? Si je réussis le concours, je vais prendre des vacances.

La conditionnelle si je réussis le concours implique que le locuteur n'est pas lauréat dans la situation d'énonciation. La condition du procès est donc présentée comme n'étant pas réalisée. Un paramètre divergent entre les deux situations étant exprimé, la sélection du FS est alors nécessaire, ce qui indique que le FS désigne un procès en rupture avec la situation d'énonciation.

39 L'emploi du FP dans ce type d'énoncé réduirait la charge hypothétique de la conditionnelle, en en faisant une condition déjà réalisée dans la situation d'énonciation (cf. ci-dessous), ce qui pour l'énoncé (4) semble incohérent.

40 La même analyse peut permettre d'expliquer l'emploi contraint du FS quand, dans des énoncés, une temporelle indique explicitement une situation différente de la situation d'énonciation comme par exemple :

(5) Au moment où tu liras ces lignes, je serai loin

*Au moment où tu vas lire ces lignes, je serai (vais être) loin (Exemple de Franckel,

1984)

41 Cet énoncé n'indique pas seulement la rupture temporelle entre deux situations mais la rupture entre l'ensemble de chaque situation. Ainsi, la situation du procès potentiel je serai présente le locuteur dans un lieu autre que celui de la situation d'énonciation. Et symétriquement, l'interlocuteur, absent du lieu de la situation d'énonciation, est présent dans ce lieu du procès potentiel tu liras. C'est cette absence de continuité entre les circonstances de la situation d'énonciation et celle du procès potentiel qui justifie de la contrainte d'emploi au FS.

42 On peut, en outre, souligner que l'alternance entre FS et FP dans des procès en série est pour le moins problématique, ce qui est un autre cas d'absence d'interchangeabilité entre les deux formes :

(6) ? Elle appellera un taxi, elle va aller à la gare et elle prendra le train.

Ces exemples tendent à montrer que FS et FP ne sont pas interchangeables. Le FS semble inadéquat quand l'énoncé souligne l'existence dans la situation d'énonciation de conditions du procès potentiel alors que le FP n'est pas admis dans des énoncés qui 
soulignent l'absence de telles conditions dans la situation d'énonciation. C'est donc que le FP indique un lien entre un procès potentiel et la situation d'énonciation quand le FS introduit une rupture par rapport à la situation d'énonciation. Selon cette analyse, la valeur sémantique du morphème a du FS ne serait donc pas la valeur du présent.

\subsection{Des tendances d'emploi qui marquent des différences sémantiques}

\section{A. La prévalence du FS en co-occurrence avec des repères temporels} fait que la forme négative était statistiquement plus fréquente avec le FS qu'avec le FP. L'étude plus récente de Fleury et Branca-Rosoff (2010) confirme cette tendance. Comment l'expliquer?

(8) Elle ne va pas venir

$\mathrm{Au} \mathrm{FP}$, la négation peut porter sur la situation potentielle en la reliant à une situation d'énonciation :

(8') Elle n'a pas eu sa correspondance. Elle ne va pas venir.

48 Mais l'analyse sémantique du FP ouvre un autre possible interprétatif de sa forme négative. En effet, la négation peut porter uniquement sur la situation d'énonciation ce qui aboutit à un paradoxe : l'adéquation des paramètres entre la situation d'énonciation et la situation du procès est niée, alors que l'existence du procès potentiel est affirmée :

(8") Elle ne va pas venir, quand même !

49 Par contre, au FS, la négation s'applique sur une seule unité. Nulle ambiguïté : le FS à la forme négative nie un procès potentiel.

(9) Elle ne viendra pas.

On peut alors penser que le choix du FS quand il s'agit de désigner simplement un procès potentiel négatif serait privilégié dans l'usage pour éviter l'ambiguité du FP négatif qui peut soit nier le procès potentiel, soit l'adéquation de la situation d'énonciation à ce procès potentiel.

\section{Proximité affective et prévalence du FP}

51 Enfin, Waught et Bahloul (1996) ont soutenu, à partir d'un corpus journalistique, que la proximité affective du locuteur avec le contenu de l'énoncé pouvait avoir une incidence sur le choix du FP. Cette analyse n'a pas été reproduite à notre connaissance mais elle nous semble séduisante. Le paramètre du locuteur fait partie intégrante de la situation d'énonciation et, à ce titre, il est vraisemblable que la prise en charge de l'énoncé par le 
locuteur puisse être un élément de continuité entre la situation d'énonciation et l'énoncé. Mais peut-il être le seul élément de continuité ? La prise de distance du locuteur va de pair, nous semble-t-il, avec la désignation de la rupture de toute une situation avec la situation d'énonciation.

De façon plus générale, l'implication du locuteur dans sa désignation du procès potentiel pourrait être exprimée par la mise en relation de la situation d'énonciation avec le procès potentiel. Toujours est-il que ce principe de choix du locuteur, correspondant à un vouloir-dire difficile à reconstituer, rend bien compte de ce que ces différences d'emploi entre FS et FP s'établissent en termes de "tendances » à interpréter beaucoup plus qu'en termes de « contraintes grammaticales ».

En définitive, ces tendances statistiques tendent à souligner une différence sémantique entre les deux futurs.

\subsection{Cas d'interchangeabilité entre le FS et le FP : réalité ou mirage?}

L'absence dans le contexte de l'énoncé, d'éléments explicites motivant le choix du locuteur entre les deux formes du futur ne signifie pas pour autant, selon nous, une absence de motivation du choix.

\section{A. Interprétation différente d'énoncés formellement équivalents}

Nous l'avons vu, certains énoncés apparemment équivalents, comme (8) elle ne va pas venir et (9) elle ne viendra pas, peuvent relever de motivations différentes allant jusqu'à dire ou nier l'existence d'un procès. Il en est de même de certains énoncés qui affichent formellement un simple échange entre FS et FP :

(10) Si elle vient, on sortira

(11) Si elle vient, on va sortir

En réalité, ces deux énoncés ne désignent pas la même relation entre les deux procès. Alors que dans l'énoncé (10) la condition si elle vient du procès potentiel est hypothétique et donc absente de la situation d'énonciation, dans l'énoncé (11), sa mise en relation avec un verbe au FP en fait une condition actualisée dans la situation d'énonciation, équivalente d'un puisqu'elle vient. Le FP tend donc à récuser le caractère hypothétique de la conditionnelle en en faisant une causale.

\section{B. Un choix entre rupture et continuité dont la motivation est peu reconstituable}

Le locuteur qui choisit de dire :

(12) Je vais nager la semaine prochaine plutôt que :

(13) Je nagerai la semaine prochaine

ne dit probablement pas la même chose. Dans le premier cas, il indique une relation entre le procès nager et la situation d'énonciation (l'habitude déjà contractée d'aller nager, la décision prise par le locuteur, l'achat d'une carte d'abonnement, etc.), alors que dans l'énoncé (13) il dit que les conditions d'accomplissement du procès ne sont pas celles de la situation d'énonciation. Dans l'énoncé (12) la semaine prochaine sert d'indicateur de temporalité du procès potentiel nager, alors que dans l'énoncé (13) la semaine prochaine participe de la rupture de la situation potentielle je nagerai en s'opposant à un maintenant. 
60 Abouda et Skrovec $(2017$ :17) soulignent que c'est ce type d'énoncés où le choix semble libre et la substitution entre les deux futurs possibles qui statistiquement conduit à une bascule de l'usage au profit du FP. Si l'on analyse que ces choix restent motivés par la prise en compte de la situation d'énonciation dans la représentation du procès potentiel, ces résultats pourraient être mis sur le compte d'une implication subjective plus grande des locuteurs francophones dans leurs énoncés.

\section{Des paramètres de la situation d'énonciation différents de ceux de la situation de locution}

61 Barbezan (2010:7) présente des exemples dans lesquels, pragmatiquement, tous les paramètres de la situation d'énonciation sont réunis mais où justement, il serait brutal, selon elle, de souligner leur présence par l'emploi du FP et où le locuteur choisit d'occulter dans son message la présence de tels indices de la réalisation du procès :

(14) Vous prendrez cette serviette après votre bain

(15) Vous terminerez ces exercices pour demain

62 Cette analyse montre bien que ce n'est pas la présence effective d'indices de la réalisation d'un procès dans une situation réelle qui est en jeu dans le choix d'un FS ou d'un FP, mais la situation d'énonciation représentée, construite par le locuteur (cf. de Vogüé, ci-dessus).

63 On le constate, la nuance est toujours difficile à percevoir en réception - réception, de toute façon, soumise aux aléas d'interprétations multiples qui brouillent l'intercompréhension des analystes - et peut-être aussi en production, si elle est établie de façon non consciente. Aussi peut-on s'interroger sur la possibilité de mettre au jour de façon systématique les critères justifiant l'emploi de FS ou de FP. Au total, même si le locuteur semble pouvoir souvent faire alterner apparemment librement l'un et l'autre, nous postulons qu'un énoncé au FS et un énoncé au FP ne sont pas équivalents, quand bien même le locuteur s'avoue incapable d'en reconstruire la justification.

L'étude des cas que nous venons d'établir rejoint bien des analyses sémantiques qui décrivent le FS comme en rupture avec le présent et le FP comme contiguë au présent, comme celle-ci de Franckel $(1984: 66)^{8}$ :

" Aller + infinitif implique une contiguité au moment de l'énonciation (que nous noterons $\mathrm{t}_{0}$ ). [...] Au contraire, le futur correspond à un aoristique, en rupture avec $t_{0}$.»

66 La différence des analyses que nous avons posées consiste en la mise en exergue d'autres paramètres que la temporalité pour traiter de la continuité ou de la rupture entre la situation d'énonciation et la situation du procès.

67 La formulation de Colmant (1975: 52) qui traite du FS comme une représentation du locuteur semble laisser ouverte la porte à ce type d'analyse :

68 "Toute la différence réside dans la façon dont le locuteur conçoit l'avenir : ou comme à l'état embryonnaire dans le présent et donc peu distinct de lui, ou comme un au-delà du présent, partant sans rapport avec lui. »

69 L'analyse des divergences et des convergences entre FS et FP a pour intérêt de mettre en exergue ces paramètres de la situation autre que la temporalité d'une part, et construits par le locuteur, d'autre part. Si cette analyse est juste, alors se limiter à la temporalité pourrait être source d'erreur, de même que se fier aux conditions réelles de la situation d'énonciation. 
70 Les analyses d'énoncés que nous avons menées nous conduisent à conclure que le FS se différencie du FP par l'absence de continuité avec la situation d'énonciation. La valeur du présent du FP ne s'appliquerait donc pas au morphème a du FS. Cependant, la comparaison entre le FP et le FS ne permet que d'approcher la valeur du morphème a dans la mesure où une unité verbale ne s'oppose pas à une composition de deux unités quand bien même cette composition s'est grammaticalisée. Comment analyser ce trait de rupture avec la situation d'énonciation : s'agit-il d'un trait sémantique ou d'un effet de sens découlant d'une valeur sémantique ? Il va s'agir à présent de replacer la concurrence FP/FS dans un cadre plus large.

\section{Analyse sémantique du FS et mise en perspective avec le FP}

\subsection{Analyse sémantique du FS effectuée par opposition avec le conditionnel}

71 La comparaison entre le FS et le FP ne permet que d'approcher la valeur du morphème a dans la mesure où une unité verbale ne s'oppose pas à un ensemble composé de deux unités. Pour cerner la valeur sémantique du morphème $\mathbf{a}$, il est nécessaire de convoquer le conditionnel qui s'oppose au FS par ce seul morphème a/ai (tu ouvriras/tu ouvrirais), avant d'examiner les conséquences de cette analyse pour le contraste FS/FP.

\section{A. Le trait « défini »}

72 FS et conditionnel ont en commun de présenter une situation potentielle - trait qui réinvestit le $\mathbf{R}$ qu'ils partagent - mais ils ne la représentent pas de la même façon.

Si on s'appuie sur une représentation ramifiée de l'avenir, comme ensemble de « mondes possibles » pour reprendre le concept développé par Martin (1981 et 1983) et Vet (1981), on peut considérer que le locuteur a le choix entre deux façons de prendre en compte cette représentation : soit en conservant l'ensemble des possibles, en ne les limitant pas, soit en circonscrivant à un seul de ces mondes possibles la désignation de l'avenir.

Selon nous, le choix du conditionnel correspond à la première option, quand celui du FS correspond à la seconde. Ainsi, le conditionnel dans :

(14) Les électeurs voteraient non

n'exclut pas l'existence d'autres situations qui rendraient d'autres procès possibles :

Les électeurs voteraient oui

Les électeurs s'abstiendraient

Les électeurs ne pourraient pas aller voter

$76 \mathrm{Au}$ conditionnel, le procès potentiel n'est pas unique, circonscrit. Le conditionnel ne réduit pas les possibles au seul procès qu'il énonce. Nous dirons que le procès est «non défini » pour indiquer cette représentation, en creux, de plusieurs procès possibles.

Par contre, le procès au FS, dans

(15) Les électeurs voteront non

exclut l'existence d'autres situations qui rendraient d'autres procès possibles. Les procès, dans :

Les électeurs voteront oui 
Les électeurs s'abstiendront

Les électeurs ne pourront pas aller voter

79

\section{la représentation de l'avenir.}

convoqué par le conditionnel pour s'y opposer. Le caractère non-défini des procès au conditionnel permet la convocation de n'importe quel paramètre de la situation d'énonciation. Le concept potentiel qui décline la non-coïncidence avec la situation d'énonciation peut alors aussi désigner un autre locuteur que celui de la situation d'énonciation : c'est ce qui explique la polyphonie d'énoncés comme " Il serait malade », la source de l'énoncé est alors indéfinie et c'est ce qui rend le procès lui-même indéfini. Il peut qualifier aussi un autre univers : il s'agit alors d'univers fictionnels comme « La caissière refuserait même l'argent des héros » construit par le narrateur dans Voyage au bout de la nuit (Céline) et dans ce cas, ce sont des circonstances autres, indéfinies qui justifient l'emploi du conditionnel.

83 Cette interprétation, parce qu'elle ne donne pas de place privilégiée à la temporalité, qui n'est qu'un paramètre de l'ensemble de la situation d'énonciation nécessairement convoquée lors de tout recours à une forme verbale, permet ainsi d'englober les «valeurs modales » du conditionnel.

\section{Le trait « défini » et l'effet de sens « catégorique »}

Le choix du FS circonscrit à un seul procès l'ensemble des procès possibles. Quand un locuteur dit Les électeurs voteront non, il désigne le procès voteront comme le seul qui puisse se produire, c'est-à-dire qu'il en assure la réalisation, quelle que soit la réalité ou sa véritable assurance que le procès voter se produise effectivement. Autrement dit, bien que le procès soit seulement possible, le locuteur le désigne de façon «catégorique » pour reprendre l'appellation de Guillaume (1929) qui l'oppose à un trait « hypothétique » du conditionnel. Nous avons souligné, par ailleurs, l'aspect paradoxal d'une telle alliance du « possible » et du « catégorique».

Or, la représentation "catégorique » du procès qui découle de ce trait " défini ", si elle permet de distinguer des procès au FS et des procès au conditionnel, n'est pas, à proprement parler, ce qui fait la différence entre le FS et le FP. En effet, ce dernier s'appuie sur sa «continuité » avec la situation d'énonciation pour affirmer le procès à venir. Aussi produit-il lui aussi des effets de sens qu'on pourrait qualifier de « catégoriques ». C'est ce qui explique les divergences sur le trait certain / incertain dont rend compte par exemple Barbazan (2010 : 4-5). 


\section{Le trait « défini » et la rupture avec la situation d'énonciation} passé simple, quand on l'oppose à l'imparfait, désigne des procès " circonscrits ", " uniques ». Le verbe au passé simple réduit à la seule situation qu'il désigne toutes les situations existantes. Autrement dit, il exclut toute situation autre, ce qui justifie, par exemple, la désignation d'une succession de procès séparés les uns des autres, quand sont juxtaposés des verbes au passé simple :

(16) Elle appela un taxi, alla à la gare et prit son train. l'existence d'autres situations avérées, que ces dernières, coïncidentes, s'inscrivent en parallèle du procès énoncé à l'imparfait (17) ou qu'elles s'inscrivent en son sein (18). Le simple énoncé Il neigeait éveille aussitôt notre curiosité car il évoque nécessairement des procès coïncidents, il contient en creux la promesse d'autres situations coexistantes :

(17) Il neigeait et tout le monde se calfeutrait chez soi.

(18) Il neigeait quand cela arriva. termes que celle entre FS et conditionnel. Encore faut-il pour cela s'extraire d'une conception purement temporelle de ces oppositions sans quoi on se heurte, par exemple, à l'impossibilité de traduire terme à terme un schéma d'incidence par le couple FS/ conditionnel :

(17') ? Il neigera quand cela arriverait.

effet, le conditionnel n'est pas le pendant « sécant » du FS dans l'avenir et les analyses qui cherchent à repérer si le FS est sécant (incident), global ou neutre sur le plan de l'incidence en analysant les relations entre les procès à l'intérieur d'un énoncé 
aboutissent à des résultats contradictoires (Rosier \& Wilmet, 2003 ; Bres, 2009). Il semble qu'en s'appliquant à la seule temporalité du procès au FS, elles réduisent la valeur du trait qui se combine avec le potentiel.

Pour nous, l'analyse demande à être menée sur l'ensemble de la situation convoquée par le verbe et le rapprochement sémantique entre FS et passé simple ne peut s'observer terme à terme dans ces mises en relation.

Pour finir, la désignation d'une situation circonscrite et unique par l'emploi d'un passé simple peut être rapportée à une valeur, celle de "défini », dont l'abstraction permet de dépasser certaines difficultés d'analyse du passé simple et de l'imparfait ${ }^{9}$. Le concept de «+/- défini » permet de désigner ce qui réinvestit en sens le morphème a/ai commun aux couples [passé simple/imparfait] et [FS/conditionnel].

6 Au total, le trait «défini » postulé à partir de l'opposition du FS avec le conditionnel permet d'expliquer la singularité du FS par rapport au FP. L'emploi d'un FS désigne une situation potentielle " définie », c'est-à-dire " circonscrite » et cette situation potentielle circonscrite, réduite à elle-même, n'entretient aucun rapport avec aucune autre situation, la situation d'énonciation n'en étant qu'une parmi d'autres.

\section{Conclusion}

En définitive, nous avons examinée ici la cohérence d'une analyse sémantique articulée à une analyse morphologique, qui postule que :

- le FP, par son trait sémantique « coïncident à la situation d'énonciation », implique une relation entre la situation d'énonciation construite par le locuteur et le procès potentiel,

9 - le FS désigne, par son trait sémantique "défini» qui se combine avec le trait «potentiel », un procès circonscrit par rapport à toute autre situation et donc à la situation d'énonciation.

- Ces deux temps seraient donc radicalement différents comme en témoignent certains emplois très tranchés mais aussi l'analyse de cas apparents d'interchangeabilité qui ne seraient que de faux semblants.

1 Cette analyse, si elle en recoupe d'autres depuis longtemps établies qui mettent en exergue la continuité ou la rupture du procès à venir avec la situation d'énonciation, en diffère d'une part en proposant une explication qui englobe la notion de rupture comme effet de sens d'un trait sémantique "défini » du FS rattachable à un morphème a, commun au passé simple, d'autre part en élargissant la désignation des temps verbaux à l'ensemble des paramètres de la situation.

En délimitant précisément ce qui sépare FS et FP, cette analyse peut sans doute apporter sa contribution à la réflexion sur un éventuel remplacement du premier par le second. En effet, elle pourrait permettre de circonscrire les observations à mener pour évaluer l'évolution des emplois. Le FS est-il en train de se spécialiser (comme le suggèrent Abouda et Skrovec, $2017: 18)$ dans des emplois devenus plus rares mais toujours nécessaires, où il désigne un univers décroché par rapport à la situation d'énonciation ? Ou est-il possible que le FP prenne peu à peu en charge ce type d'énoncés et devienne polysémique, à l'instar du passé composé ? Il s'agirait alors d'orienter les recherches de corpus vers les situations qui inscrivent le procès dans la rupture avec la situation d'énonciation de façon à évaluer si l'emploi du FP dans des énoncés comme "ben alors, qu'est-ce que ça fera ces 
jeunes-là quand les parents vont plus être là ?» (Abouda \& Skrovec, 2017:9) relèvent de l'erreur, d'un lien implicite avec la situation d'énonciation ou d'une polysémie émergente de cette forme.

\section{BIBLIOGRAPHIE}

ABOUDA, L., SKROVEC, M., 2015, « Du rapport entre formes synthétique et analytique du futur. Étude de la variable modale dans un corpus oral micro-diachronique ", Revue de sémantique et pragmatique, Presses de l'Université d'Orléans, p. 35-57.

ABOUDA, L., SKROVEC, M., 2017, « Du rapport micro-diachronique futur simple/futur périphrastique en français moderne. Étude des variables temporelles et aspectuelles ", Corela, HS 21, <http://corela.revues.org/4804>

ALLAIRE, S., 1984, «L'infinitif a-t-il un sujet ? ", Tétralogiques, 1, p. 49-93.

BARBAZAN, M., 2010, « Comment choisir le meilleur des futurs ? Contraintes et libertés d'usage pour le futur simple et le futur composé », L'Information grammaticale 127, p. 1-9.

BARCELO, G. J., 2007, « Le(s) futur(s) dans les langues romanes : évolution linéaire ou cyclique ? ", Cahiers Chronos 16, Amsterdam, New-York, p. 47-62.

CAMUSSI-NI, M.-A., 2006, Analyse formelle et conceptuelle des formes verbales du français contemporain : À la croisée du passé simple et de l'imparfait, du futur et du conditionnel, les concepts '+-potentiel' et '+/- défini', Thèse de doctorat, Université Rennes 2.

CAMUSSI-NI, M.-A., 2009, « De la présence du présent dans le futur simple », Faits de langue 33, p. 19-26.

CAMUSSI-NI, M.-A., 2010, « La permutabilité de certaines formes verbales met-elle à mal la relation entre les domaines de la morphologie et de la sémantique? » Liens Linguistiques, Etude sur la combinatoire et la hiérarchie des composants, éd. Alvarez Castro C., Bango de la Campa F., Donaire L., Peter Lang, p. 227-240.

COLMANT, G., 1975, « Un nouveau futur en français? », Le langage et l'homme 29, p. 51-54.

CONFAIS, J.-P. , 1990, Temps, mode, aspect, Toulouse, Presses Universitaires du Mirail.

CULIOLI, A., 1999, Pour une linguistique de l'énonciation, T. 2, Paris, Ophrys.

DE VOGÜÉ, S., 1991, « La transitivité comme question théorique : querelle entre la Théorie des Positions de J.C. Milner et la Théorie des Opérations prédicatives et Enonciatives d'A. Culioli », Linx 24, p. 37-65.

DE VOGÜÉ, S., 2000, « Calcul des valeurs d'un énoncé 'au présent' », Travaux de linguistique 40, p. 31-54.

DE VOGÜÉ, S., 2006, « Qu'est-ce qu'un verbe ? », in Constructions verbales et production de sens », D. Lebaud, C. Paulin, K. Ploog (eds), Presses Universitaires de Franche-Comté, p. 43-62.

FLEISHMAN, S., 1983, « From pragmatics to grammar : diachronic reflections on complex pasts and futures in Romance », Lingua 60, p. 183-214. 
FLEURY, S., BRANCA, S., 2010, « Une expérience de collaboration entre linguiste et spécialiste de TAL : L'exploitation du corpus CFPP 2000 en vue d'un travail sur l'alternance Futur simple / Futur périphrastique ", Cahiers AFLS 16(1), p. 63-98.

FRANCKEL, J.-J., 1984, « Futur 'simple' et futur 'proche' », Le Français dans le monde 182, p. 65-70.

GAGNEPAIN, J., 1982, Du Vouloir-dire, Paris, Livre et communication.

GUILLAUME, G., 1929, Temps et verbe. Théorie des aspects, des modes et des temps, Paris, Champion.

JEANJEAN, C., 1988, «Le futur simple et le futur périphrastique en français parlé. Étude distributionnelle », dans BLANCHE-BENVENISTE, C., CHERVEL, A., GROSS, M. (éds.), Hommage à la mémoire de Jean Stéfanini, Aix, Presses de l'université de Provence, p. 235-258.

LAURENDEAU, P., 2000, «L'alternance futur simple et futur périphrastique : une hypothèse modale », Verbum 22-3, p. 277-292.

MARTIN, R. 1981, «Le futur linguistique : temps linéaire ou temps ramifié ? », Langages 64, p. 81-92.

RÉMY-GIRAUD, S., 1988, «Les grilles de Procuste. Description comparée de l'infinitif en français, grec ancien, allemand, anglais et arabe », dans Rémy-Giraud, S. (éd.), L'Infinitif, Lyon, Presses Universitaires de Lyon, p. 11-68.

ROSIER, L., WILMET, M., 2003, « La concordance des temps revisitée, ou de la concordance à la convergence ", Langue Française 138, 97-110.

SUNDELL, L.-G., 1991, Le temps futur en français moderne, Almqvist \&Wiksell International, Acta Universitatis Upsaliensis, Stockholm.

URIEN, J.-Y., 1984, Marque et immanence dans la théorie du signe. Tétralogiques 1, p. 7-32.

VET, C., 1981, « La notion de 'monde possible' et le système temporel », Langages 64, p. 109-124.

VET, C., 1985, « Univers de discours et univers d'énonciation : les temps du passé et du futur », Langue Française 67, p. 38-58.

VET, C., 1993, « Conditions d'emploi et interprétations des temps futurs du français », Verbum 4/1993, p. 71-84.

VET, C., 2007, « The descriptive inadequacy of Reichenbach's tense system: A new proposal », Cahiers Chronos 17, p. 7-26.

VETTERS, C., LIÈRE, A., 2009, «Quand une périphrase verbale devient temps verbal : le cas d'aller + infinitif $»$, Faits de langue 33, p. 27-36.

WAUGH, R., BAHLOUL, M., 1996, « La différence entre le futur simple et le futur périphrastique dans le discours journalistique », Modèles linguistiques 33, XVII-1, p. 19-36.

WILMET, M., 2003, Grammaire critique du français, Bruxelles, Duculot.

\section{NOTES}

1. Nous symbolisons par ai, le morphème réalisé par la série ( $a i, i)$ et par $\mathbf{a}$, celui réalisé par la série (ai, a, Ø). De même, $\mathbf{R}$ permet de désigner commodément l'ensemble de ses allomorphes.

2. Allomorphes du R au FS et conditionnel : /r/, /ər/, /ir/ ; à l'infinitif :/r/, /ir/, /e/, /war/.

3. Cette analyse prend en compte les zéros, c'est-à-dire, les absences qui s'opposent significativement à un matériau. En effet, comme le souligne Urien (1984 : 13), « la marque n'est 
pas le matériau, c'est-à-dire la séquence de phonèmes considérée en elle-même, mais de la variation de matériau, de la différence entre séquences, qui atteste le franchissement d'une frontière sémiologique, quelle que soit la modalité de cette frontière structurale : valeur lexicale, textuelle, paradigmatique ou syntaxique, qui sont les composantes d'une même capacité de grammaticalité ». Le zéro n'est pas une marque, pas plus que ne l'est le matériau auquel il s'oppose, mais l'opposition significative entre la présence et l'absence de matériau constitue la marque.

4. Allaire souligne aussi «le parallèle qui s'impose entre l'opération qui donne un sujet [à l'infinitif] et celle qui lui attache des valeurs temporelles - valeurs toujours liées à celles de son environnement, verbal et non verbal, et toujours explicables par la contestation de ses silences syntaxiques. » (Allaire, $1984: 91$ )

5. Pour de Vogüé, la notion d'« éventualité » participe à la définition du verbe : il peut référer « à des éventualités, c'est-à-dire à ce qui peut être ou ne pas être le cas » (2006:52).

6. De Vogüé (2000) montre que la temporalité n'est pas centrale pour définir le présent mais elle postule, quant à elle, une absence de morphème spécifique et rattache la variabilité des sens attribué au présent à une analyse fine des différents types de configurations énonciatives.

7. À noter que ce ne sont pas les termes attention et tiens qui rendraient la sélection du FS improbable, mais le fait qu'ils désignent souvent des paramètres d'une situation d'énonciation qui conditionnent un procès potentiel. De fait, ils peuvent introduire un verbe au FS quand le locuteur présente un procès en rupture avec la situation d'énonciation (Attention, tu te feras écraser un jour ; tiens, il pleuvra demain).

8. On peut citer aussi Jeanjean (1988), Confais (1990), Wilmet (2007).

9. Cf. Camussi-Ni (2010)

\section{RÉSUMÉS}

Le débat sur la concurrence entre le futur simple et le futur périphrastique réapparait périodiquement, et en particulier l'idée d'une grammaticalisation aboutissant au remplacement du premier par le second. De fait, les études de fréquences d'emploi soulignent la domination du futur périphrastique sur le futur simple. Est-ce pour autant le signe de la disparition de ce dernier? La question est conditionnée par celle d'une équivalence sémantique des deux futurs. Cet article réexamine cette question de l'interchangeabilité des deux formes futures pour tenter une explication de l'acceptabilité ou de la non-acceptabilité de certains emplois de chacune de ces formes. L'hypothèse avancée ici est que le réinvestissement sémantique des oppositions morphologiques entre futur simple et futur périphrastique impliquerait une opposition sémantique entre les deux formes qui en limiterait l'interchangeabilité. Cette hypothèse, qui s'inscrit dans le prolongement de travaux sur l'opposition entre le futur simple et le conditionnel (Faits de langue 33), conduit à proposer une analyse du futur simple comme combinant un trait sémantique commun au futur périphrastique, le trait « potentiel» de l'infinitif, et un autre trait, divergent, le trait "défini ». Elle s'inscrit aussi dans le cadre d'une conception du verbe qui inclut, au-delà du paramètre temporel, la totalité de la situation d'énonciation.

The debate about the competition between the simple future tense and the periphrastic future is regularly relaunched, mainly by the idea that the latter is replacing the former through a grammaticalization process. Corpus analysis does indeed highlight the supremacy of the 
periphrastic future. Yet does it mean that the simple future will disappear? In fact, this question stems from the notion that the two future tenses are semantically equivalent. This article reviews the question of the interchangeability of the two tenses by focusing on the acceptability and non-acceptability of some of their uses. Our hypothesis is that the interchangeability of the two tenses may be limited by a semantical contrast inherent to the morphology of the simple and the periphrastic futures. This hypothesis builds on our work on the contrast between the simple future and the conditional (Faits de langue 33) and leads us to analyse the simple future as the combination of two features, a semantic feature also possessed by the periphrastic future, namely the 'potential' feature of the infinitive, and another different one, the 'definite' feature. Our hypothesis is part of a verb approach encompassing not only the temporal parameter but also the situation of enunciation as a whole.

INDEX

Mots-clés : temps futur simple, futur périphrastique

Keywords : simple future tense, periphrastic future

\section{AUTEUR}

MARIE-ARMELLE CAMUSSI-NI

CELLAM, Université Rennes 2 